FORMATLON Formation emploi

Revue française de sciences sociales

150 | avril-juin 2020

Former aux "petits" métiers : regards internationaux

\title{
Des socialisations professionnelles tramées par des logiques scolaires : les CAP coiffure et métiers de l'automobile en lycée
}

When academic process shapes professional socialisation: hairdressing and automotive trades vocational education in high school

Berufliche Sozialisation im Schulkontext : das CAP für die Friseur- und Kfz-

Branche in der Berufsschule

Socializaciones profesionales marcadas por la lógica escolar : los CAP peluquería y oficios automotrices en la escuela secundaria

\section{Sophie Denave, Fanny Renard et Camille Noûs}

\section{OpenEdition}

Journals

Édition électronique

URL : https://journals.openedition.org/formationemploi/8238

DOI : 10.4000/formationemploi.8238

ISSN : 2107-0946

Éditeur

La Documentation française

Édition imprimée

Date de publication : 8 juillet 2020

Pagination : 145-165

ISSN : 0759-6340

Référence électronique

Sophie Denave, Fanny Renard et Camille Noûs, « Des socialisations professionnelles tramées par des logiques scolaires : les CAP coiffure et métiers de l'automobile en lycée », Formation emploi [En ligne], 150 | avril-juin 2020, mis en ligne le 08 juillet 2020, consulté le 08 janvier 2022. URL : http:// journals.openedition.org/formationemploi/8238; DOI : https://doi.org/10.4000/formationemploi.8238 


\title{
Des socialisations professionnelles tramées par des logiques scolaires: les CAP coiffure et métiers de l'automobile en lycée
}

\begin{abstract}
Sophie Denave
Maîtresse de conférences en sociologie, Université Lumière Lyon 2, Centre Max Weber (UMR 5283), équipe MEPS (Modes, espaces et processus de socialisation). Sociologie de la socialisation, des parcours scolaires et professionnels et des classes populaires

FANNY RenARD Maîtresse de conférences en sociologie, Université de Poitiers, GRESCO (Groupe de recherches sociologiques sur les sociétés contemporaines), EA 3815, Sociologie de la socialisation et de la scolarisation des classes populaires

Camille Noûs

Membre du Laboratoire Cogitamus
\end{abstract}

Résumé

Des socialisations professionnelles tramées par des logiques scolaires : les CAP coiffure et métiers de l'automobile en lycée

Cet article étudie la spécificité de la socialisation professionnelle dans les formations au CAP (Certificat d'aptitude professionnelle) coiffure et métiers de l'automobile, effectuées sous statut scolaire. Il montre que des logiques proprement scolaires façonnent tant les modalités de la socialisation (mode scolaire de transmission des savoirs et savoir-faire, socialisation anticipatrice et scolarisation des stages) que les appropriations de ces formations par les lycéens. Du fait du recrutement différencié de ces spécialités, les lycéens des métiers de l'automobile se montrent plus rétifs à l'ordre scolaire que les lycéennes de la spécialité coiffure.

Mots clés : CAP - certificat d'aptitude professionnelle, enseignement techniqueprofessionnel, socialisation professionnelle, coiffeur, métier de l'automobile, orientation scolaire-professionnelle, lycée professionnel, transmission des savoirs, savoir professionnel, stage de formation, représentation de la formation 
Abstract

When academic process shapes professional socialisation: hairdressing and automotive trades vocational education in high school

This article studies the specificity of professional socialisation in CAP (level 1 vocational qualification) for hairdressing and automotive trades vocational education in high school. It shows that strictly academic process shapes both the modes of socialisation (academic transmission of knowledge and know-how, anticipatory socialisation and work placements overseen by high schools) and the appropriation of these training courses by high school students. Because of a different selection in these two specialisations, the girls studying hairdressing are far less restive than the boys studying automotive trades.

Keywords: certificate of vocational aptitude, technical \& vocational education, professional socialisation, hairdresser, occupation in the car industry, school guidance $\&$ vocational guidance, vocational upper secondary school, knowledge transmission, professional knowledge, traineeship, perception of training

Journal of Economic Literature: I 21, M 53

Traduction : auteures et Carole Couegnas.

\section{Introduction}

En France, la formation aux métiers ouvriers et employés s'effectue non seulement par l'apprentissage en entreprise, mais aussi par la voie scolaire en lycée professionnel. Cet article vise à mettre en évidence comment l'école s'y prend pour préparer à ces métiers et comment les élèves s'emparent des formations proposées. Autrement dit, il s'agit d'étudier la spécificité de la socialisation professionnelle à l'œuvre dans ces formations préparées par la voie scolaire.

À cette fin, deux spécialités sont comparées : respectivement les CAP coiffure et métiers de l'automobile (maintenance de véhicules automobiles - MV - et réparation des carrosseries - RC). Historiquement, ces CAP se préparent par la voie de l'apprentissage (Moreau, 2003). Salariés, les jeunes sont alors principalement formés par un maître d'apprentissage en entreprise (trois semaines par mois) ${ }^{1}$. Ils y sont directement confrontés aux enjeux de production et à la pénibilité du travail (Denave, Renard, 2019). L'apprentissage reste dominant pour ces spécialités ${ }^{2}$, mais la voie scolaire $s^{\prime}$ est

1. Les apprentis suivent une formation scolarisée en Centre de formation d'apprentis (CFA) une semaine par mois.

2. Il conserve le monopole de la formation au brevet professionnel de coiffure et l'Association nationale pour la formation automobile exhibe les meilleurs taux d'emploi des jeunes diplômés de CAP sortant d'apprentissage : en 2018, sept mois après la fin de leur formation, 59,4\% des apprentis en CAP MV option 
développée et la part des jeunes formés au lycée n’est plus négligeable : $44 \%$ des jeunes préparant un CAP coiffure et $30 \%$ de ceux préparant un CAP métiers de l'automobile sont lycéen $s^{3}$ et principalement formés par des enseignants. Sur deux ans de formation, les lycéens ne passent que douze semaines en entreprise lors des stages. Coexistent ainsi deux formations distinctes pour un même diplôme. La comparaison de ces deux spécialités permet d'observer que les modalités de la socialisation tiennent fortement à la voie de formation et à son mode de recrutement.

Dans un contexte de valorisation de l'alternance (Agulhon, 2000), ces formations professionnelles ont notamment vu augmenter la durée des stages et la place de l'entreprise dans la formation. Avec l'institutionnalisation des relations entre l'école et l'entreprise, se sont également imposés le "modèle pédagogique de compétences" et le rapprochement des référentiels de diplômes et d'emplois (Tanguy, 2005). Renvoyant de surcroît à la division sexuelle du travail, ces formations accueillent un public non mixte (Mosconi, 1983) : la part des garçons est de $98.6 \%$ en CAP maintenance de véhicules automobiles et de $98.1 \%$ en réparation des carrosseries, tandis que la part des filles est de $93.3 \%$ en CAP coiffure ${ }^{4}$. À cet égard, le monde du travail participe bel et bien à la socialisation professionnelle en lycée, mais celle-ci ne s'y réduit pas. Les formations orchestrent en effet une socialisation professionnelle marquée par des logiques proprement scolaires. Nous montrons, dans une première partie, que les modalités de la socialisation s'inscrivent dans ce que Guy Vincent a nommé "la forme scolaire " (Vincent, Lahire, Thin, 1994) : un enseignement dispensé par des personnels dédiés et dans des relations pédagogiques séparées des autres relations sociales ; un enseignement rationalisé de savoirs et pratiques qui ont conquis leur logique par l'écrit et s'acquièrent par le biais d'exercices "conçus aux seules fins d'apprentissage "; un enseignement au cours duquel s'intériorise "la soumission à des règles impersonnelles " 5 . D'une part, bien que situées en bas de la hiérarchie de l'enseignement secondaire (Palheta, 2012) et recrutant principalement des jeunes issus des classes populaires aux parcours scolaires caractérisés par des redoublements, un passage par les classes atypiques du collège $\left(3^{\mathrm{e}}\right.$ prépa-pro, Section d'enseignement général et professionnel adapté (SEGPA), Maison familiale rurale, etc.), ou encore des ruptures scolaires, ces formations professionnelles sont tramées par un mode scolaire de transmission des savoirs et savoir-faire. D'autre

"véhicules particuliers " sont en emploi, contre 31,4\% des lycéens et en CAP RC, 51,1\% des apprentis contre 25,2\% des lycéens (ANFA, 2019).

3. Bases élèves et apprentis, France métropolitaine, 2012, Banque Centrale de Pilotage, ministère de l'Éducation nationale.

4. Bases élèves, France métropolitaine, 2012, Banque Centrale de Pilotage, ministère de l'Éducation nationale. À ce titre, nous emploierons le masculin pour les lycéens des métiers de l'automobile et le féminin pour les lycéennes de coiffure.

5. Devenu " dominant " par sa diffusion et sa légitimation, ce mode de socialisation ne se retrouve plus seulement à l'école, mais aussi dans de nombreuses familles, chez les animateurs et travailleurs sociaux, etc., et ne porte pas seulement sur les savoirs d'école (lecture, écriture, etc.). 
part, une "socialisation anticipatrice " est à l'œuvre dans ces formations (Darmon, 2013). En effet, les jeunes n'expérimentent pas le travail dans les conditions salariales mais, au sein de relations pédagogiques, ils incorporent des savoir-faire et intériorisent des manières d'être attendues dans les secteurs professionnels visés.

Dans la seconde partie, nous analysons la manière dont des logiques scolaires, via le mode de recrutement dans les spécialités ${ }^{6}$ et les parcours scolaires des élèves, modèlent les appropriations de ces formations par les jeunes. Les lycéens des métiers de l'automobile se montrent plus rétifs que les lycéennes de coiffure au mode scolaire de transmission et à la socialisation anticipatrice. En lycée professionnel, les métiers de l'automobile réservent plutôt l'accès en CAP aux élèves en difficulté scolaire, provenant de SEGPA ou ne pouvant accéder à une seconde générale ou professionnelle, tandis que la coiffure retient les candidats au niveau scolaire le plus élevé relativement aux élèves des autres $\mathrm{CAP}^{7}$ (Denave, Renard, 2017).

\section{Encadré 1. Méthodologie}

Cet article s'appuie sur une recherche qui interroge les processus d'orientation scolaire vers les CAP (Certificat d'aptitude professionnelle) respectivement coiffure et métiers de l'automobile, et la socialisation professionnelle qui s'y déroule $\left(^{*}\right)$. Cette recherche mobilise à la fois des données quantitatives et ethnographiques relatives aux jeunes fréquentant ces CAP. L'enquête a été conduite au sein de deux centres de formation d'apprentis (CFA) et de deux lycées professionnels (LP) dans les académies de Lyon et Poitiers. Nous mobilisons ici le matériau produit dans les LP : des entretiens avec dix enseignants et responsables de formation, des observations des cours, de la vie des établissements et de visites de stages. Nous avons aussi réalisé des entretiens approfondis auprès de jeunes en 1 re ou 2de année de CAP, portant sur les dimensions scolaires, familiales et amicales de leur trajectoire. Conformément à la composition sexuée de ces CAP, nous avons rencontré essentiellement des garçons dans les CAP des métiers de l'automobile (une seule fille sur les vingt élèves mécaniciens et carrossiers) et une majorité de filles en CAP coiffure (trois garçons sur les seize élèves). Les lycéens interrogés sont presque tous issus des classes populaires stabilisées, avec des parents en emploi. Les pères travaillent majoritairement comme ouvriers, artisans ou commerçants et les mères comme employées.

${ }^{*}$ ) : La recherche a été financée, dans le cadre de l'appel à projets «L'égalité des chances à l'école », par la Direction de l'évaluation, de la prospective et de la performance (DEPP) du ministère de l'Éducation nationale, de l'Enseignement supérieur et de la Recherche, par le Commissariat général à l'égalité des territoires (CGET, anciennement Acsé) et par le Défenseur des droits.

6. Le lycée professionnel recrute sur critères scolaires et assure une place à tous les élèves sous obligation scolaire, mais pas toujours dans la spécialité demandée. Son type de sélection dépend de la structuration des diplômes de chaque spécialité (CAP-Brevet professionnel en coiffure contre CAP et/ou Bac professionnel pour les métiers de l'automobile).

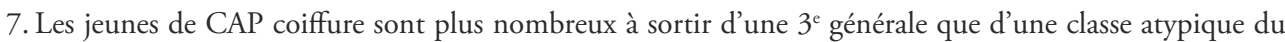
collège par exemple. 


\section{Une socialisation professionnelle hors des lieux ou des relations de travail}

Au cours du $\mathrm{Xx}^{\mathrm{e}}$ siècle, les formations professionnelles du secondaire ont été à la fois scolarisées et utilisées comme "outil de résolution du problème de la scolarisation de masse dans le collège unique [...] voie de remédiation de l'échec scolaire " (Tanguy, 2013, p. 31). Paradoxalement, elles exposent des élèves au parcours antérieur heurté, souvent recalés de l'enseignement général, à un mode scolaire de transmission des savoirs et savoir-faire professionnels, à une socialisation anticipatrice et à une découverte scolarisée de l'univers professionnel.

\subsection{Un mode scolaire de transmission des savoirs et savoir-faire}

Les savoirs et savoir-faire enseignés sont effectivement distincts en coiffure et dans les métiers de l'automobile. Mais, d'une spécialité à l'autre, les savoirs et savoir-faire professionnels font l'objet, en lycée, d'un même mode scolaire de transmission.

La préparation d'un CAP en lycée professionnel s'effectue principalement en établissement scolaire, lieu dédié à l'enseignement. Les enseignements dits professionnels (technologiques et pratiques) occupent une position centrale dans une formation qui prépare à un métier. Centrale, d'abord parce qu'ils sont chargés d'une dimension symbolique forte. Ils sont immédiatement identifiés comme permettant l'acquisition du métier et sont dispensés par d'anciens professionnels ayant exercé plus ou moins longtemps avant de devenir enseignants (ils ont le plus souvent passé le Concours d'accès au corps des professeurs de lycée professionnel ${ }^{8}$ ). Mais cette centralité se repère aussi aux coefficients aux examens et au volume horaire : les coefficients des épreuves professionnelles sont bien supérieurs à ceux des épreuves d'enseignement général ${ }^{9}$; les enseignements professionnels (enseignement pratique, en atelier ou salon pédagogique - douze heures hebdomadaires -, et technologique, en classe) représentent plus de la moitié de la formation en établissement (dix-huit heures sur trente-deux ou trente heures hebdomadaires). Cette place centrale se manifeste encore par l'investissement économique qu'elle requiert via l'achat de matériels spécialisés ${ }^{10}$, les outils.

8. Dans le lycée d'enquête, la plupart des enseignants de coiffure ont suivi une formation en LP ; même celle qui l'a effectuée en apprentissage se montre acquise au mode scolaire de transmission.

9. Les matières d'enseignement général ont un coefficient sept en MV, six en RC et six en coiffure, tandis que les épreuves professionnelles ont un coefficient quatorze en MV, dix-sept en RC et seize en coiffure.

10. En coiffure, l'achat du « trousseau " (terme utilisé par les enseignants lors d'une réunion d'information auprès de parents d'élèves de $3^{\text {e }}$ : ciseaux, brosses, peignes...) est pris en charge à $50 \%$ par la Région, mais les familles doivent payer l'autre moitié, soit environ $200 €$. Dans les métiers de l'automobile, une caisse à outils est fournie à chaque lycéen de la région lors de son inscription en CAP. Il lui reste à charge de se munir d'un bleu de travail et d'une paire de chaussures de sécurité (entre 80 et $160 €$ ). 
Pour autant, contre un ethnocentrisme qui tendrait à considérer que cet enseignement diffère fondamentalement des enseignements dits généraux en raison de leur caractère «manuel» engageant le corps (Faure, 2000), il convient de montrer qu'il relève aussi d'un mode scolaire de transmission.

L'enseignement des savoirs et savoir-faire professionnels s'inscrit dans une programmation et une progression. En coiffure, par exemple, la réalisation d'une couleur est au programme du CAP, mais "les mèches " figurent dans celui de BP (brevet professionnel). Une même logique de "progression dans la difficulté " (enseignant MV) apparaît en maintenance de véhicules automobiles, allant des "choses les plus simples ", c'est-à-dire les entretiens courants (vidanges, remplacement des filtres, etc.) en $1^{\text {re }}$ année, aux "choses un peu plus techniques" comme l'embrayage, l'année suivante. Les savoirs et savoir-faire sont aussi décomposés pour être acquis par le biais d'exercices répétés jusqu’à ce que les savoir-faire soient incorporés. Lors d'un cours sur les boucles, les élèves de coiffure les réalisent sur l'ensemble de la tête, avant de la rincer et de recommencer les boucles à nouveau. Les élèves en coiffure s'exercent sur des " têtes " en silicone, et sur des tôles, des voitures d'atelier ${ }^{11}$, voire des moteurs extraits des voitures, pour les élèves en maintenance de véhicules automobiles :

"Des fois, y’a des tâches difficiles à faire sur des véhicules et les exécuter direct sur les véhicules, c'est galère. On apprend d'abord à le faire sur un moteur libre, où y'a rien autour, et après on le fait sur le moteur. Mais sur la voiture, c'est pas pareil [...] là y’a la carrosserie et tout le truc autour. " (Clément, $1^{\text {re }}$ année CAP MV)

En atelier ou en salon pédagogique, le temps, libéré des enjeux de production, est dédié à la formation et l'activité se distingue de celle qui s'exerce en entreprise. À l'occasion d'un cours de piquetage en coiffure, technique au cours de laquelle on peut se blesser (les élèves apprennent à " travailler " la frange en tenant les ciseaux en direction des doigts), les élèves, appliqués, vont particulièrement doucement. Lorsque la sonnerie de fin de cours retentit, ils ne sont pas arrivés au séchage et au coiffage de la chevelure. L'enseignant s'en désole - "en salon, ce n'est pas possible de laisser une cliente avec une coupe et un séchage non finis "-, mais il laisse partir les élèves qu'il n'a pas particulièrement pressés durant le cours, plus attentif à ce qu'ils s'appliquent sur l'acquisition d'une nouvelle technique.

Enfin, en plus de disposer d'un temps long pour la pratique, les élèves, en petits groupes (les salons pédagogiques regroupent quinze élèves), disposent d'un enseignant qu'ils peuvent solliciter, plutôt que de collègues affairés au bon déroulement du travail. Cet enseignant veille encore à constituer une culture de métier (Zarca, 1988). Une ensei-

11. Les voitures d'atelier sont des voitures dont le seul usage est d'être réparées par les élèves. Elles ne roulent pas hors du lycée professionnel. 
gnante de coiffure prend plaisir à présenter les noms des outils et à expliquer leur histoire :

"Les outils, le matériel, [...] j'adore [enseigner]... ben mon côté un peu brocante tu sais? [...] [je] leur dis "Ben vous savez autrefois... Pourquoi les ciseaux? Ben parce que normalement, on part de l'idée qu'on a un ciseau, c'est comme les menuisiers... "J'adore ça moi, leur faire mon speech... Et puis, qu'elles sortent en ayant compris qu'en réalité on dit des ciseaux, parce qu'un ciseau, c'est quand il n'y en a que la moitié!» (Entretien, Mme Meyer, responsable des enseignements coiffure)

Surtout, enseignements généraux, technologiques et pratiques (savoirs et savoir-faire pourrait-on dire) sont fortement articulés. Les enseignements de mathématiques et de français-histoire-géographie, par exemple, nourrissent les savoirs professionnels :

"En mécanique, y’a la fréquence des moteurs et en maths, on a fait comment calculer la fréquence de rotation, donc ben c'est en lien, au moins on peut relier, on peut calculer et tout. En français, on a travaillé sur l'histoire des ouvriers de France en mécanique auto, c'est lié. Enfin, voilà, nos profs essaient toujours de lier [...] le plus avec notre métier [...] ça nous apprend, pour certains qui connaissent pas, comment a évolué l'automobile. " (Clément, $1^{\text {re }}$ année CAP MV)

En coiffure, la maîtrise de la coloration s'appuie sur les enseignements technologiques et pratiques, mais aussi de biologie. En cours de pratique, les enseignants font référence à des enseignements technologiques et exigent leur maitrise de la part des élèves pour une bonne acquisition des savoir-faire. Mme Barreau demande à une élève de lui réciter des produits. Comme elle échoue, l'enseignante commente :

"Fixateur? Eau oxygénée. Révélateur? Ammoniaque. Pour casser les ponts cystines? Acide. Je vais te le faire copier 20 fois, c'est le b.a.-ba" (Journal de terrain(JT), LP coiffure).

Outre la connaissance et la qualification des produits et de leurs effets, les gestes professionnels sont explicités et justifiés. En cours pratique, une enseignante de coiffure reprend ainsi un élève à qui elle a demandé de décrire son geste :

"Explique les gestes avec les mots professionnels ("je tiens la mèche-témoin à 90 degré") c'est ce qui sera évalué".

Observant les élèves travailler, les enseignants décrivent les gestes à réaliser et s'assurent de l'intériorisation d'un discours sur la pratique :

"Il faut mettre des mots sur les gestes, contrairement à ce que reçoivent les apprentis. Généralement, dans les salons, on dit "fais ça", mais il faut mettre les mots et pas seulement du visuel, la méthode est importante pour vous. " (Mme Barreau en atelier, JT, LP coiffure) 
Les savoir-faire enseignés sont objectivés. Avant ou après leurs exercices, les élèves se voient présenter une fiche technique. En coiffure, les élèves apprennent à considérer une tête et une chevelure sous forme schématisée (sont représentées les séparations entre les mèches à travailler). En cours de permanente, ils doivent dessiner sur une feuille des bigoudis en fonction des mises en plis à réaliser (classique ou en quinconce). En cours de technologie, les aspirants mécaniciens apprennent, à partir de schémas dessinés au tableau, non seulement les noms des pièces automobiles, mais aussi les gestes, décomposés, à effectuer, ou encore à tenir la posture scolaire :

"C'est toujours bien de montrer avant les pièces et tout, de voir comment c'est avant d'y aller, plutôt que d'aller directement sur le moteur et pas savoir comment faire [...] ’̀ un certain moment, faut savoir, comme on dit, se calmer et être patient devant une table, même si on aimerait être devant un véhicule et direct travailler. " (Clément, $1^{\text {re }}$ année CAP MV)

En atelier, avant de s'affairer sur les voitures, les lycéens du secteur automobile doivent aussi remplir des "sujets d'étude ", c'est-à-dire répondre à des questions sur un document dactylographié de quatre à cinq pages. Ces exercices permettent de connaître en détail les pièces automobiles et les outils :

"À chaque fois qu'il faut voir une voiture, faut remplir plein de papiers. Comme dans un garage aussi, mais... là c'est qu'il faut faire des recherches dans des livres. Faut beaucoup sinstruire sur les pièces. Vraiment savoir tout sur tout. " (Sasha, $2^{\text {de }}$ année CAP MV)

Enfin, ils utilisent des revues techniques qui décrivent chaque étape d'un processus de réparation. Savoir ainsi démonter et remonter les pièces automobiles en suivant une fiche technique les forme à la réparation de tout type de modèle automobile. Les formateurs de lycée jugent indispensable, et spécifique à une formation scolaire, l'enseignement d'une "mémoire procédurale " (Grignon, 2015) :

"Nous on essaie de leur donner une méthode globale. Le jour où ils tombent sur quelque chose qu'ils n'ont jamais vu, ben, ils prennent le document et ils font. Par contre, au garage, on leur montre, [les stagiaires] singent les gestes et ils recommencent. Par contre, sils tombent sur un autre vébicule où c'est un peu différent, ben, ils ont pas l'information."

Dans les établissements d'enquête, les élèves avaient de surcroît plusieurs enseignants de pratique rendant possible la confrontation à des manières différentes de réaliser les gestes professionnels et d'appréhender des techniques variées ${ }^{12}$.

Ainsi, en atelier, il s'agit de ne pas limiter l'acquisition aux savoir-faire, de ne pas enfermer la pratique "dans les limites étroites d'un procédé ou d'un geste " appris sommairement par l'imitation (Brucy, 2013, p. 17), ni dans les habitudes individuelles d'un maître d'apprentissage.

12. En coiffure, comme en automobile, les enseignants se répartissent les domaines d'apprentissage (moteur, électricité, liaison-sol et maintenance; coupes femmes, coupes hommes, mises en forme, coloration). 


\section{2 Être sensibilisé aux conditions de travail sans les expérimenter : une socialisation anticipatrice}

La division sexuelle du travail commandant l'organisation scolaire, les élèves des deux spécialités évoluent dans des classes composées très majoritairement de filles ou de garçons. Ils et elles sont ainsi familiarisé.e.s avec des configurations relationnelles non mixtes et les conditions d'enseignement des savoirs et savoir-faire professionnels laissent place à la conformation à des normes de genre en vigueur dans les secteurs professionnels visés (Lamamra, 2016).

Mais contrairement aux apprentis, les lycéens sont principalement formés dans l'enceinte du lycée, dans des conditions et relations scolaires ou pédagogiques qui se distinguent des conditions et relations salariales. Ces dernières ne sont pas expérimentées par les lycéens, même si elles imprègnent les justifications et discours professoraux comme futurs probables. Les élèves y sont préparés par le biais d'une socialisation professionnelle anticipatrice qui, relativement au mode scolaire de transmission des savoirs et savoir-faire, relève moins d'un enseignement systématique et rationalisé.

Hors du contexte professionnel, les lycéens sont globalement préservés des conditions de travail et d'emploi. Ils sont soumis à des exigences temporelles. Mais comme lycéens, ils n'ont pas les mêmes contraintes horaires que les salariés et bénéficient davantage de temps de repos (récréations, vacances scolaires). S’ils doivent justifier leurs absences ou les retards répétés, c'est auprès de la vie scolaire ; ils n'ont pas besoin d'un arrêt de travail et ne risquent pas la rupture de contrat. Au pire, la menace de l'échec au diplôme est brandie.

Le rythme de réalisation des tâches est aussi différent et n'est pas dicté par les contraintes de productivité. En plus d'être guidé par les temps impartis pour l'examen, parfois très éloignés des durées prévues par les barèmes constructeurs de temps de main-d'œuvre, ce rythme vise la bonne acquisition des gestes professionnels et l'apprentissage du suivi des procédures de sécurité, qui ralentissent la cadence. Il en va de même de la pénibilité physique du travail dans les métiers de l'automobile et de la coiffure (Denave, Renard, 2019). Si les lycéens éprouvent celle suscitée par certains gestes professionnels (répétitions, postures, port de charges lourdes...), ils n'y sont confrontés que douze heures par semaine, contre trente-cinq heures pour les apprentis. En $1^{\text {re }}$ année de CAP coiffure, les lycéennes sont encore préservées des produits toxiques et utilisent du dentifrice ou du talc à la place des colorations.

Par ailleurs, sans être insérés dans des relations salariales, les lycéens sont conduits, au gré d'injonctions professorales, à adopter des attitudes conformes au statut et à l'univers professionnel visés par le diplôme préparé et se voient reprocher, par leurs enseignants, des manières d'être qui y dérogent. Les lycéens des métiers de l'automobile et de la coiffure doivent manifester une attitude active, se montrer affairés à la tâche. Lors d'un cours de carrosserie, l'enseignant reprend un élève s'apprêtant à travailler en posi- 
tion assise sur une voiture. Désignée comme attitude non professionnelle, cette posture est aussi présentée comme sanctionnable en garage. L'enseignant précise à l'enquêtrice : "On peut travailler assis, mais ça se fait pas dans les garages car on passe pour un fainéant" et il insiste sur la nécessité d' "un savoir-être irréprochable " pour travailler dans le secteur automobile.

Les lycéens sont aussi enjoints d'obéir à leur futur patron ou de feindre la soumission (Kergoat, 2006). Préparant un départ en stage à l'occasion d'un cours de coiffure, une enseignante invite ses élèves à trouver "normal » que la tutrice, mais aussi cheffe d'entreprise, refuse que ses clientes soient coiffées par des débutantes (au regard du prix des services et des impératifs de fidélisation de la clientèle ; Desprat, 2017). Elle les incite aussi à ne pas relever les vexations dont les professionnelles font l'objet de la part de la clientèle (Barbier, 2012) et à se soumettre à ses souhaits tout en travaillant sur des têtes en silicone. À des propos d'élèves dépréciant la permanente ("c'est moche, c'est vieux"), l'enseignante réagit vivement et ordonne de maîtriser cette technique : "Les petites vieilles qui veulent une permanente, ce sont celles qui vont faire tourner votre salon!"

Enfin, pour les lycéens des deux spécialités sont valorisées des présentations de soi distinctes, propres aux univers professionnels non mixtes visés. Comme dans les garages, le port des bleus de travail et des chaussures de sécurité est obligatoire dans le lycée automobile. En outre, des manières d'être spécifiques sont transmises. En carrosserie, par exemple, "On est des gueulards, on rote, on pète » précise un enseignant. Avec le bruit des outils dans les ateliers, il faut effectivement parler fort pour se faire entendre, mais c'est aussi la revendication d'une certaine forme de masculinité virile propre aux professionnels de l'automobile qui est mise en scène et transmise aux élèves. À l'inverse, les élèves de coiffure se voient incitées à policer leur langage pour se préparer aux échanges avec la clientèle (dans les couloirs, une enseignante reprend des élèves qui nous disent «Bjour M'dames" au lieu de "Bonjour Mesdames") et à travailler leur apparence, sans qu'il y ait une codification stricte ${ }^{13}$ : les nouvelles tenues vestimentaires et coupes ou coiffures des élèves sont ainsi fréquemment relevées, et de la sorte, encouragées, par les enseignants en salons pédagogiques qui livrent aussi des astuces et ficelles : acheter dans des magasins proposant des vêtements peu onéreux, mais aux styles recherchés (Zara); équiper de semelles ses chaussures de ville (pour éviter les baskets sans avoir mal au dos), etc.

Ainsi, la formation en lycée propose bel et bien une socialisation professionnelle en orchestrant l'incorporation de gestes du métier et l'intériorisation d'injonctions concernant l'ajustement des manières d'être à l'emploi dans les secteurs visés. Cependant, cette socialisation, au sein de relations pédagogiques, est anticipatrice dans la mesure où les élèves n'expérimentent pas la condition salariale. Ils sont préservés, dans une certaine mesure, de la pénibilité physique et morale du travail.

13. Hormis l'interdiction du port de décolletés plongeants ou de mini-jupes et la forte injonction à s'apprêter, il n'y a pas d'indications très précises sur les tenues vestimentaires, le maquillage et la coiffure. 


\subsection{Les stages : une découverte scolarisée de l'univers professionnel}

Parallèlement à la revalorisation de l'alternance, les périodes de formation en milieu professionnel (PFMP) ont vu leur importance augmenter. La durée de stage est ainsi passée de six à douze semaines pour le CAP coiffure et les CAP métiers de l'automobile. Les PFMP ont aussi vu leurs prérogatives se modifier. Certains des éléments du programme de formation leur sont désormais dévolus :

"Ça s'est fait avec les professionnels hein : y a eu une table ronde, des réunions de travail. Et on leur a dit "Voilà, à partir de maintenant, vous allez avoir les jeunes six semaines par an [...] y a des choses qu'on n'aura plus le temps de leur faire [faire au lycée]. [...]" Et on a un petit peu essayé de voir ensemble... ce qu'ils pouvaient, eux, leur enseigner. En réalité, on est tombé d'accord sur le fait que, eux, ils n'avaient pas le temps à la formation. Mais par contre, qu'ils pouvaient consolider... les bases que nous on leur inculquait ici. [...] Avant [2007 $\left.{ }^{14}\right]$ non, c'était un stage de formation, mais... il venait renforcer ce qu'on leur apprenait nous! Mais les professionnels n'avaient pas en charge de leur apprendre des choses. " (Mme Meyer)

Si l'enseignement des savoir-faire se réalise en lycée et en entreprise, officiellement, les entreprises ont la prérogative de l'enseignement de "savoir-être " (autonomie dans le travail, relation à la clientèle, en coiffure, "la communication ", etc.). Les PFMP deviennent encore un des lieux d'évaluation des compétences juvéniles : la pratique professionnelle est évaluée pour moitié lors des PFMP, le reste, au lycée, lors de contrôles en cours de formation. De fait, les enseignants des lycées professionnels n'ont pas le monopole de la formation et sont même placés dans une relation de dépendance visà-vis des tuteurs de stage. Ils doivent trouver des entreprises qui acceptent de prendre en charge leurs élèves, au risque de rendre impossibles la préparation et la validation du CAP.

Or, la formation en entreprise se caractérise fondamentalement par le fait d'apprendre le métier tout en le pratiquant aux côtés d'autres professionnels (Suteau, 2012). Aux dires des élèves et des enseignants, les tuteurs privilégient une formation par voirfaire, en montrant les gestes et en utilisant peu les revues techniques, par exemple. Ils escomptent des stagiaires qu'ils prennent place dans l'organisation du travail et, ne maîtrisant pas encore les savoir-faire, accomplissent les petites tâches (ménage, rangement, shampoing, ou pneumatique pour le CAP métiers de l'automobile). Moins personnels dédiés à l'enseignement que professionnels ayant en charge le bon fonctionnement de l'entreprise, ils peuvent être objectivement conduits à faire primer les impératifs de production sur ceux de la formation (Moreau, 2003). Ils familiarisent les lycéens avec l'univers professionnel, le métier (Zarca, 1988).

14. Réforme du CAP coiffure. 
Toutefois, les évolutions ayant accru la place de l'entreprise dans la formation ont aussi conduit à transformer l'entreprise en entreprise formatrice. Désormais plus explicitement soumise au référentiel de formation, elle est d'une certaine manière scolarisée.

En plus d'être soustraites au lien de subordination, les périodes de formation en milieu professionnel demeurent sous contrôle professoral : les enseignants participent à l'élaboration d'un cahier des charges des stages. Ils peuvent décider de ne plus confier de stagiaires aux entreprises qui ne le respectent pas. L'activité des jeunes en entreprise, et par ricochet celle des tuteurs, est encadrée et guidée par un livret de formation. Aussi, en coiffure, les lycéennes de $1^{\text {re }}$ année de CAP travaillent finalement peu sur la clientèle. Même en stage, elles peuvent s'entraîner sur des têtes en silicone (permanente ou mise en plis) ou, si elles peuvent solliciter leur entourage, sur des modèles (coupe, brushing, couleur). Les professionnels doivent remplir le livret de formation et y consigner leur évaluation des acquisitions de leurs stagiaires. Mais ils sont accompagnés, dans cette pratique, par le personnel enseignant qui les aide à transformer en notes les appréciations émises sur les stagiaires, le barème étant fixé par l'Éducation nationale :

"Evaluation en entreprise. [...] On va demander au professionnel de faire une synthèse de toutes les activités que le jeune a faites. Et d'évaluer... la réussite de ces activités [...] mais de... décortiquer un petit peu chaque geste. [...] Et... le professionnel va donc mettre des plus et des moins. Avec ces différents indicateurs d'évaluation. Nous, quand on va passer [en visite de stage], on va l'aider le professionnel et on va transposer ses plus et ses moins en points. [...] par exemple, pour "choix adapté des outils et des accessoires", le professionnel sil a mis plus, on lui dira "Et ben pour cette ligne-là, le barème, c'est 1,5." (Mme Meyer)

Les enseignants peinent cependant parfois à amener les tuteurs de stage à se défaire de leur propre classification qui privilégie les savoir-être aux dépens des savoirs répertoriés dans la grille d'évaluation des stages (niveau d'activité, habileté, qualité du travail, esprit d'initiative, etc.) :

"Pour la note: [reprenant les termes d'un tuteur] "[cet élève] est gentil, il est bien, on va lui mettre 15 à lui, parce qu'il est gentil, il est à l'heure, il est poli”. " (Enseignant de mécanique)

Le personnel enseignant intervient encore lorsque les élèves expriment des difficultés sur leur lieu de stage (relations conflictuelles, attribution du sale boulot et moindre place accordée à la formation, exigence de savoir-faire non encore enseignés ou imposition d'un temps de réalisation des tâches trop court...) et va parfois jusqu'à autoriser un changement d'entreprise.

Ainsi, la familiarisation des lycéens avec l'univers professionnel est encore fortement scolarisée et occupe une période brève, bien qu'allongée, par rapport à la formation qui se réalise en lycée. La socialisation professionnelle est d'une certaine manière encore anticipatrice. Même lors des périodes de formation en milieu professionnel, elle ne se fait pas sous contrat de travail et reste encadrée par des relations pédagogiques. 


\section{Les appropriations juvéniles des formations sont sous-tendues par des logiques scolaires}

Interrogés sur la formation, les lycéens valorisent davantage les enseignements professionnels que les enseignements technologiques ou généraux.

Dans les deux spécialités, les lycéens jugent positivement les ateliers pédagogiques dans lesquels ils estiment apprendre leur métier. Sans que cela se réalise toujours aisément, ils apprécient le fait d'acquérir des gestes techniques et un vocabulaire spécialisé, de manier les outils du métier auquel ils sont préparés. Ils valorisent les relations plus personnalisées avec les enseignants d'ateliers. Ces ateliers offrent par ailleurs des respirations dans une formation en établissement scolaire, en autorisant notamment les mouvements et déplacements.

Cette préférence générale pour les ateliers n'empêche pas certaines critiques. Les jeunes éprouvent des difficultés à s'engager dans les apprentissages en atelier qui s'éloignent des conditions de travail. Dans les métiers de l'automobile comme en coiffure, les lycéens se plaignent de la systématicité de la formation (s'entraîner à des mises en plis " démodées " et peu pratiquées dans les salons) et du côté répétitif et artificiel des exercices proposés.

L'appropriation, par les lycéens, du mode scolaire de transmission des savoirs et savoirfaire et de la socialisation anticipatrice varie néanmoins selon la spécialité de formation et les conditions d'accès à celle-ci. Les élèves de coiffure adhèrent au mode scolaire de socialisation, tandis que les élèves du secteur automobile se montrent plus rétifs.

\subsection{Des élèves de coiffure plus ajustées au mode scolaire de socialisation}

Du fait d'une socialisation sexuée plus consonante avec les attentes scolaires et d'un recrutement relativement sélectif (choix restreint de spécialités « féminines » et nombre de places limité en CAP coiffure), les filles préparant le CAP coiffure au lycée semblent relativement ajustées au mode scolaire de transmission des savoir-faire et à la socialisation anticipatrice.

La majorité des élèves de coiffure rencontrées apprécient de découvrir les conditions de vie lycéennes et plusieurs participent aux diverses activités proposées par l'établissement scolaire : groupe de musique, cours de zumba, cours de basket, sorties sportives. En $3^{\mathrm{e}}$, certaines n'ont pas cherché à signer un contrat d'apprentissage pour préparer leur CAP ("Je me voyais pas travailler... à 14-15 ans "; "J'hésitais à aller au contact de la clientèle. Donc je préférais venir en... lycée professionnel ") et elles semblent ne pas regretter de reporter à plus tard l'expérimentation de la violence redoutée des relations de travail et de la «domination servicielle» (Jeantet, 2003). 
Elles valorisent une acquisition progressive du métier, la technicité de l'enseignement professionnel, et une expérience, circonscrite et sous contrôle professoral, de la pénibilité physique et morale. Lise, qui souhaitait initialement préparer son CAP en apprentissage et a essuyé trois refus dans sa recherche de patron, s'est laissée convaincre, par la conseillère d'orientation-psychologue, de ses «capacités » à entrer en lycée professionnel et de l'intérêt de la voie scolaire : on peut y apprendre un métier progressivement, sans être immédiatement confronté aux difficultés de la vie active. En fin de $1^{\text {re }}$ année, elle a fait siens ces arguments. Entre deux cours, elle explique à l'enquêtrice que le lycée lui permet de s'habituer à être debout progressivement (trois heures par jour) " en apprentissage, on est debout toute la journée dès le début. " Cette valorisation d'une socialisation anticipatrice ne recoupe pas nécessairement l'aisance avec laquelle les lycéennes s'approprient les savoir-faire professionnels. Ainsi, Julie est décrite par l'une de ses enseignantes comme ayant eu "des moufles à la place des mains " lors des premiers mois de formation. Les difficultés que rencontre Gaëtan dans la réalisation d'une permanente (lenteur) n'entachent pas son appréciation de la spécialité et du mode d'enseignement des savoir-faire :

"Je maîtrise bien, mais... je vais pas assez vite, c'est tout. Mais quand j'irai vite, comme la $2^{e}$ année qu'on a eue [en cours] [...] Elle nous a montré, elle a fait une permanente en 30 minutes! [...] Du coup, on a fait "L'année prochaine on fera pareil" parce qu'elle nous a dit "Ouais, maintenant, j'aime bien la permanente... c'est trop sympa." Du coup, on verra bien l'année prochaine hein. " (Gaëtan, $1^{\text {re }}$ année CAP coiffure)

Ayant fait l'objet d'une sélection scolaire relative pour accéder à une formation professionnelle attractive (Arrighi, Gasquet, 2010), ces élèves se sentent " rescapées ", y compris celles qui souhaitaient initialement préparer un CAP par apprentissage ou une autre spécialité, comme l'esthétique-parfumerie. Aussi, elles se plient plus volontiers à l'enseignement proposé et reconnaissent l'autorité pédagogique des enseignants. Peu de retards et d'absences ont été observés lors des salons pédagogiques. Alors qu'elle ne peut manier les ciseaux suite à une blessure au poignet, Aline participe tout de même au cours de pratique et réalise du cannage sur une chevelure (tressage spécifique) à la place du piquetage. Il n'est pas rare de voir, durant les pauses (récréations...), les élèves assises dans les couloirs, classeurs ouverts sur les genoux.

Les élèves respectent le plus souvent les consignes (y compris lors de séances laborieuses et répétitives) et expriment a minima leur lassitude ou difficultés par des soupirs. S’inquiétant de la perception de son attitude (nombreuses plaintes) par l'enquêtrice, Océane dit à une camarade, lors d'une séance de permanente : "Elle va croire que je n'aime pas la coiffure ». Les lycéennes mettent en œuvre des formes discrètes de contestation de l'ordre scolaire qui rompent peu le fil de la communication pédagogique (Depoilly, 2014). Elles manifestent encore une intériorisation des jugements scolaires : elles paraissent ainsi heureuses lorsque les enseignants valorisent leur maîtrise de savoirfaire (une élève sourit au retour de son enseignante sur son brushing : "Bien, avec peu de cheveux, tu as réussi à faire une Mamie Nova, tu as bien fait gonfler, très belles mèches, 
regardez toutes ") et vexées et affectées lorsque les enseignants pointent la non-maîtrise de savoir-faire ou jugent leurs comportements non conformes à ceux de coiffeuses. À une élève qui a mal aligné ses bigoudis, l'enseignante enjoint :

"Il faut s'entraîner à la maison une heure par jour". Lise pleure, se fait consoler par deux camarades. " (JT, LP coiffure)

Au lycée, les élèves prennent encore plaisir à travailler leur tenue vestimentaire et leur apparence qui leur assurent une certaine popularité au sein de leurs groupes de pairs. Plusieurs lycéennes racontent avec fierté qu'elles se sont coupé les cheveux depuis leur arrivée au lycée. Alors qu'elles se singularisaient par une très longue chevelure, elles se distinguent désormais par une coupe travaillée. Nombreuses aussi sont les élèves qui arborent des tenues remarquables répondant aux critères professoraux et lycéens de la recherche vestimentaire. Aline est félicitée par son enseignant pour la cohérence de son look "rockeuse " (une coupe courte, dégradée sur les côtés, cheveux blond platine sur les pointes et noirs à la racine, rouge à lèvres vermillon, gilet et pantalon en jeans, chemisier blanc en mousseline, col clouté). Des élèves, qui discutent "onglerie " (deux couches de vernis, puis une couche de durcisseur pour empêcher que le vernis ne s'écaille) après avoir terminé et réussi leur exercice de brushing, ne se font pas reprendre par leur enseignante pourtant peu tolérante aux bavardages lycéens. Dans les salons pédagogiques, les lycéennes s'essaient avec leurs camarades à des types de conversation possibles en salon (même si les préoccupations et sociabilités juvéniles reprennent parfois le dessus).

Les lycéennes apprécient le plus souvent les stages réalisés (sociabilités informelles avec des coiffeuses, fréquentation d'un univers professionnel, essais de savoir-faire, gratifications de la clientèle - pourboire...). Cependant, plusieurs évoquent aussi les tensions suscitées par cette expérience : la difficulté de l'isolement par rapport aux pairs, la timidité, la crainte de commettre des erreurs sur la clientèle ou sur leur modèle.

Certaines expriment aussi les difficultés rencontrées avec les tutrices de stage. Océane est ainsi remontée contre la tutrice de son premier stage qui a critiqué son attitude lors de la visite de l'enseignante ou qui corrigeait certaines de ses pratiques (" elle me faisait tout le temps des réflexions comme quoi jétais pas attentive »). Ces tensions sont exacerbées dans la mesure où, dès la $1^{\text {re }}$ année de CAP, les élèves de coiffure envisagent leurs stages comme de longues périodes d'essai en vue de décrocher un contrat d'apprentissage pour préparer le brevet professionnel :

"Le salon dans lequel je vais aller... [stage 2] cherche une BP, voilà. Et donc en gros, ils comptent un petit peu sur moi apparemment pour me tester, pour voir si... ce serait possible. [...] Là c'est pour ça, y a un peu plus de stress, parce que y a plus d'enjeux. Mais... faut prendre confiance! [rire] .» (Priscillia, $1^{\text {re }}$ année CAP coiffure)

De surcroît, l'expérience de stages et la fréquentation de coiffeuses ont fait toucher du doigt aux lycéennes les difficultés à exercer le métier durant une longue période (péni- 
bilité physique et morale, faible salaire, rares congés...). Certaines construisent même le projet de devenir enseignante de coiffure après quelques années dans un salon.

Ces différents indices suggèrent le plus grand ajustement à l'institution scolaire et à la socialisation professionnelle anticipatrice des lycéennes de coiffure que des lycéens des métiers de l'automobile.

\subsection{Des élèves des métiers de l'automobile plus rétifs à l'ordre scolaire}

Les jeunes des métiers de l'automobile éprouvent des difficultés à s'engager dans les apprentissages en atelier qui s'éloignent des conditions du monde professionnel. Ce sont surtout ceux qui entretiennent une "affiliation faible au métier" (Moreau, 2010) qui résistent le plus à cette forme d'enseignement.

L’orientation imposée justifie en partie ce rapport au métier : les uns ont été inscrits en réparation des carrosseries alors qu'ils envisageaient plutôt une formation en maintenance automobile; d'autres regrettent de ne pas avoir trouvé de patron pour suivre la formation par apprentissage ou d'avoir été écartés d'une $2^{\text {de }}$ générale ou professionnelle.

Tout en appréciant davantage les enseignements des disciplines professionnelles que les autres matières, les lycéens cherchent souvent à s'extraire du travail en atelier en étant absents, en se rendant fréquemment aux toilettes ou en prolongeant le nettoyage des voitures (occasion de flâner, de rigoler, de s'arroser).

Dans les salles de cours et les ateliers pédagogiques, les relations d'autorité sont encore celles qui prévalent dans l'institution scolaire et les lycéens des métiers de l'automobile activent des formes de contestation constituées au gré d'un parcours heurté. "Ils sont immatures. Ils sont très gamins, ils vont se mettre à crier on sait pas pourquoi " déplore ainsi un enseignant de mécanique. De même, ses collègues font état de nombreux problèmes de comportement et de passages d'élèves en conseil de discipline. Critiquées par les enseignants, ces attitudes sont néanmoins relativement tolérées dans la mesure où elles questionnent plus les comportements attendus que les savoir-faire techniques enseignés. Les enseignants considèrent leurs élèves comme des lycéens à encadrer, voire à recadrer.

La plupart des lycéens se plaignent du côté répétitif et artificiel des exercices proposés au lycée. Par exemple, ils n'apprécient pas de s'exercer à la découpe de tôle par la fabrication de petits objets comme des cendriers. Les lycéens critiquent aussi l'utilisation des voitures d'ateliers qu'ils distinguent des voitures clients sur lesquelles il est plus intéressant de travailler :

"Je préfere les voitures clients parce que c'est du travail... On sait que c'est du travail quion va rendre à une personne, donc c'est mieux [...] On sait qu'on va rendre une voiture, au moins, le client il vous dit merci. Tandis que les voitures d'atelier, la plupart du temps, c'est 
des voitures, elles démarrent pas ou elles roulent jamais, donc même si on les répare mal, on sait que ça va rien changer. " (Clément, $1^{\text {re }}$ année CAP MV)

L'utilisation systématique des revues suscite des difficultés chez les élèves qui se plaignent de cette étape obligée avant le travail pratique sur les voitures. Un enseignant de mécanique constate :

\section{"[Les élèves] ont la documentation constructeur. Et c'est notre plus gros souci d'ailleurs."}

Confrontés au rythme rapide de l'exécution de tâches seulement lors des périodes de stage et ne bénéficiant pas des avantages de la condition salariale, au lycée, ils profitent cependant du fait de ne pas subir une position subalterne à laquelle ils auraient pourtant consenti s'ils étaient devenus apprentis. Non rémunérés, les lycéens refusent de porter les stigmates de la position d'ouvrier, d'où, par exemple, le rejet du bleu de travail :

"J'avais pas envie qu'on me voie comme ça, j'avais envie qu'on me voie normal. [...] Ça fait prisonnier, ça fait travailleur... soit je travaille avec une tenue et je suis payé et je fais ça toute la journée, soit... je l'enlève et voilà. » (Keny, $1^{\mathrm{re}}$ année CAP MV)

La question du salaire, ou plutôt de son absence, revient de façon récurrente dans les entretiens menés auprès des lycéens. Il leur paraît difficilement acceptable de travailler dans des ateliers de réparation automobile, c'est-à-dire dans des conditions proches des conditions de travail, sans être rémunérés. Ils ont le sentiment de ne pas bénéficier de ce qui fait la raison d'être de l'apprentissage d'un métier. Ce n'est pas sans rappeler le rapport classique des classes populaires au travail, qui le voient d'abord comme un moyen de subvenir à leurs besoins, contrairement aux travailleurs des classes moyennes ou supérieures davantage en quête d'épanouissement personnel (Baudelot \& al., 2003).

Les lycéens mécaniciens ou carrossiers, " recalés de l'apprentissage » et souvent inscrits dans des spécialités ou à un niveau de formation ne correspondant pas à leur premier vœu $^{15}$, ne s'identifient donc pas à des ouvriers, mais à des élèves rétifs à l'ordre scolaire. Ils se disent le plus souvent ravis d'aller en stage, ne serait-ce que pour sortir de l'enceinte du lycée et se retrouver dans un univers professionnel. Mais ils entretiennent un rapport ambivalent aux savoirs et au mode de transmission auxquels ils sont confrontés dans les garages. Ils peuvent tout à la fois apprécier d'être initiés à de nouveaux savoirfaire et d'être à distance de l'écrit en n'utilisant pas les revues techniques, et reprocher aux maîtres d'apprentissage de ne pas expliciter suffisamment les gestes professionnels :

"Là [dans le garage] ils expliquent pas. Là, ils font voir, font montrer, c'est à nous de poser des questions. Mais pas tout le temps car après, je le saoule. " (Elhadj, $1^{\text {re }}$ année CAP MV)

15. La formation au CAP en lycée professionnel correspond au premier vœu d'orientation de seulement six des vingt lycéens des métiers de l'automobile interrogés : neuf auraient préféré être en apprentissage ; quatre aspiraient à d'autres spécialités (pâtisserie, conducteur routier, informatique); un regrette de ne pas avoir été accepté en seconde générale. 
L'isolement par rapport aux camarades de classe et la crainte de commettre des erreurs sur les voitures pèsent aussi sur les aspirants des métiers de l'automobile. Ainsi, Medrick ( $2^{\text {de }}$ année CAP MV) se sent plus en insécurité au garage "parce que si tu fais quelque chose, si tu déchires le pneu et tout après... [cela peut générer des accidents] ", tandis qu'au lycée le "prof est toujours avec toi ". D’autres élèves ont éprouvé des difficultés à travailler sous le regard des clients. C'est le cas de Donald qui se dit stressé en présence du client : "L'émotion que t'as quand le client il est là, tu peux pas travailler bien (Rires)". Les lycéens sont enfin nombreux à reprocher la pression quant à la maîtrise des gestes, le rythme de travail plus intense qu'au lycée :

"[Au lycée], on a quatre heures. C'est bon quatre heures pour un démarreur. On a l'habitude d'y aller cool. Mais quand on a l'habitude d'y aller cool et qu'en stage, où à l'heure c'est payé, ben là... on déguste. " (Sandra, MVA, $2^{\text {de }}$ année)

Les lycéens contestent aussi leur assignation à des tâches répétitives, comme la pneumatique ou les vidanges, voire le fait d'être relégués à l'exécution du " sale boulot " (laver le garage, porter seul des caisses lourdes à la poubelle, etc.). Un cas extrême est celui de Kwalis ( $1^{\text {re }}$ année CAP MV) qui a très mal vécu sa période de stage :

"Ha moi là-bas, ça m’a saoulé. Ha moi je retourne plus à Audi, c’est bon, c’est terminé [...] C'est-à-dire ça [te] prend comme des chiens, franchement"

Enquêtrice : "Ouais t’as l'impression que t’as été traité comme un "chien" ?"

"Voilà, ils me disaient... Attends, je faisais déjà un travail, je faisais de l'aspirateur et l'autre il me disait "vas-y, fais vite, la voiture elle est pour midi". Ho, doucement! J'suis sur une voiture, c'est bon [...]. M'ont rien appris. J'ai appris juste... J'ai fait les vidanges que j'ai apprises ici [au lycée]. Mais là-bas, que nettoyage, poubelles, c'est tout"

E. : "Ha t’as fait que du nettoyage "

"C'est tout. Ça m’a saoulé [...] J'ai dit "Mais monsieur, je suis venu pour quoi là ? Je suis venu pour faire de l'atelier ou je suis venu pour laver?" Il me dit "Tais-toi et lave par terre". Tu parles comme ça toi ? J'ai appelé mon père... Mon père il m'a dit "Tu te tais et tu le fais." "

Si l'envie est bien réelle de partir en stage, les lycéens sont prévenus par les enseignants que ce "sera plus dur" (Malory, $1^{\text {re }}$ année CAP RC). Et s'ils portent un regard globalement positif sur cette période, c'est aussi parce qu'ils savent que le stage est temporaire : ils retrouveront rapidement des conditions pédagogiques de travail et les sociabilités amicales au lycée. 


\section{Conclusion}

La valorisation actuelle de l'entreprise formatrice et de l'alternance va de pair avec la dépendance originelle des formations professionnelles au monde du travail. Cependant, une enquête de terrain confrontant les points de vue enseignants et lycéens et croisant entretiens et observations de classe souligne la place qu'y prend l'École. La comparaison de deux spécialités préparant au CAP par la voie scolaire montre que les formations en lycée offrent une socialisation professionnelle largement tramée par des logiques scolaires et distincte de celle portée par l'Apprentissage, bien que celui-ci ait été aussi scolarisé. Au sein de relations pédagogiques, ces formations s'effectuent selon un mode scolaire de transmission. Elles permettent l'incorporation de gestes professionnels et l'intériorisation d'injonctions visant l'ajustement des manières d'être à l'emploi dans les garages ou les salons de coiffure. Elles préservent, dans une certaine mesure, les lycéens de la pénibilité physique et morale du travail.

De plus, la comparaison de ces deux spécialités met en évidence que les appropriations de ces modalités de socialisation, par les élèves, sont elles aussi produites par des logiques scolaires. En effet, les appropriations juvéniles découlent, pour partie, de la socialisation sexuée différenciée des filles et des garçons qui les prépare inégalement au mode scolaire de transmission (Baudelot, Establet, 1992) et «à l'évidence du travail» (Moreau, 2000). L'enquête pointe que ces appropriations juvéniles sont également liées aux parcours scolaires antérieurs et à la plus ou moins grande résistance ou conformation des élèves au mode scolaire de transmission, ainsi qu'aux recrutements distincts selon les spécialités et voies de formation (Denave, Renard, 2017). Les lycéens des métiers de l'automobile se montrent rétifs à l'ordre scolaire. N'ayant pu accéder à l'apprentissage, ils ne se projettent pas dans la position d'ouvrier, dont ils ne tirent pas immédiatement les avantages (salaire, statut professionnel), et résistent à la socialisation professionnelle anticipatrice à laquelle ils sont confrontés. Les lycéennes de la spécialité coiffure s'ajustent davantage au mode scolaire de transmission et apprécient d'être préservées des conditions salariales subalternes que subissent les apprenties. L'alternance et le mode pratique de transmission des savoirs dans des conditions salariales apparaissent ainsi ne pas représenter une solution universelle à la formation professionnelle.

\section{Bibliographie}

Agulhon C. (2000), "L'alternance : une notion polymorphe, des enjeux et des pratiques segmentés ", Revue française de pédagogie, $\mathrm{n}^{\circ}$ 131, pp. 55-63.

ANFA (2019), "Formation des services de l'automobile : forte progression des effectifs en alternance ", Autofocus. Les études de l'observatoire de l'ANFA, n 78, 8 p.

Arrighi J.-J., Gasquet C. (2010), « Orientation et affectation : la sélection dans l'enseignement professionnel du second degré », Formation Emploi, n 109, pp. 99-112. 
Barbier P. (2012), "Contrainte relationnelle et résistance au travail. Les vendeurs des grands magasins ", Sociétés contemporaines, $n^{\circ} 86$, pp. 31-57.

Baudelot C., Gollac M. (dir.) (2003), Travailler pour être heureux ? Le bonheur et le travail en France, Paris, Fayard, 351 p.

Baudelot C., Establet R. (1992), Allez les filles!, Paris, Seuil, 24 p.

Brucy G. (2013), "L'apprentissage ou... les apprentissages ? ”, Revue française de pédagogie, $\mathrm{n}^{\circ} 183$, pp. 15-26.

Darmon M. (2013), Classes préparatoires. La fabrication d'une jeunesse dominante, Paris, La Découverte, 317 p.

Denave S., Renard F. (2017), "L'orientation en CAP métiers de l'automobile et coiffure. Entre élaboration d'aspirations et conditions d'affectation ", Éducation \& Formations, $\mathrm{n}^{\circ}$ 93, pp. 43-66.

Denave S., Renard F. (2019), "Des corps en apprentissage. Effets de classe et de genre dans les métiers de l'automobile et de la coiffure ", Nouvelles questions féministes, 38/2, pp. 68-84.

Depoilly S. (2014), Filles et garçons au lycée pro, raport à l'école et rapport de genre, Rennes, Presses universitaires de Rennes, 221 p.

Desprat D. (2017), "Qu'est-ce qu'on vous fait aujourd'bui?" Un ethos professionnel des coiffeurs. Entre travail émotionnel, relation de service et dispositions genrées et de classe, thèse de doctorat en sociologie, Université Paris Nanterre, 433 p.

Faure S. (2000), «Dire et (d')écrire les pratiques de danse. Opposition entre pratiques discursives et non discursives ", Cahiers Internationaux de Sociologie, CVIII, pp. 161-178.

Grignon C. (2015), " Point de vue : bachelier ou professionnel ? ", Formation Emploi, $\mathrm{n}^{\circ} 131$, pp. 163-168.

Jeantet A. (2003), " "À votre service !" La relation de service comme rapport social ", Sociologie du travail, $\mathrm{n}^{\circ}$ 45, pp. 191-209.

Kergoat P. (2006), « De l'indocilité au travail d'une fraction des jeunesses populaires. Les apprentis et la culture ouvrière ", Sociologie du travail, n 48, pp. 545-560.

Lamamra N. (2016), Le genre de l'apprentissage, l'apprentissage du genre. Quand les arrêts prématurés révèlent les logiques à l'ouvre en formation professionnelle initiale, Zurich et Genève, Éditions Seismo, 296 p.

Moreau G. (2000), «Les faux semblables de l'apprentissage », Travail, genre et sociétés, $\mathrm{n}^{\circ} 3$, pp. 67-86.

Moreau G. (2003), Le monde apprenti, Paris, La Dispute, 273 p. 
Moreau G. (2010), « Devenir mécanicien. Affiliation et désaffiliation des apprentis aux métiers de la mécanique automobile », Swiss Journal of Sociology, n 36, pp. 73-90.

Mosconi N. (1983), "Rapports entre division sexuelle du travail et inégalités des chances entre les sexes à l'école ", Revue française de pédagogie, nº 62, pp. 41-50.

Palheta U. (2012), La domination scolaire. Sociologie de l'enseignement professionnel et de son public, Paris, Presses universitaires de France, 354 p.

Suteau M. (2012), "Les artisans et le CAP : une conversion tardive (1920-2000) ", Revue française de pédagogie, $\mathrm{n}^{\circ} 180$, pp. 43-52.

Tanguy L. (2005), "De l'éducation à la formation : quelles réformes ? ", Éducation \& Sociétés, $\mathrm{n}^{\circ} 16$, pp. 99-122.

Tanguy L. (2013), "Apprentissage en entreprise et formation professionnelle en école : une mise en perspective des années 1950 aux années 1990 ", Revue française de pédagogie, $\mathrm{n}^{\circ} 183$, pp. 27-38.

Vincent G., Lahire B., Thin D. (1994), "Sur l'histoire et la théorie de la forme scolaire ", in Vincent G. (éd.), L'éducation prisonnière de la forme scolaire? Scolarisation et socialisation dans les sociétés industrielles, Lyon, Presses Universitaires de Lyon, pp. 11-48.

Zarca B. (1988), "Identité de métier et identité artisanale », Revue française de sociologie, $\mathrm{n}^{\circ} 29$, pp. 247-273. 